\title{
Le BMS est sur la bonne voie!
}

\section{Bruno Kesseli}

Dr méd. et lic. phil., rédacteur en chef

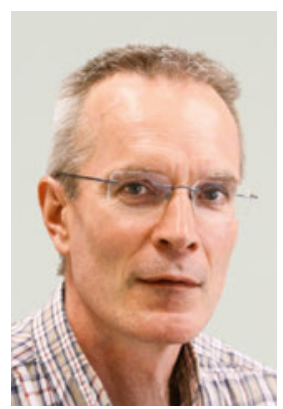

Les Editions Médicales Suisses EMH ont eu 20 ans en 2017 et sont donc à peu près sorties de la phase tempétueuse de l'adolescence. Pour autant, la flotte EMH ne vogue pas en eaux calmes, loin de là.

Cette métaphore un peu bancale a été choisie volontairement, car cet anniversaire a donné lieu à une surenchère de descriptions imagées de la situation générale. Le président du conseil d'administration Hans Kurt, dont l'article sur l'anniversaire figure dans ce numéro [1], a évoqué "Des nuages sombres et du ciel bleu» [2] lors de la réunion 2017 des EMH avec les délégués des sociétés médicales. Il faisait référence d'une part aux défis à venir liés au financement de revues indépendantes de haute qualité et d'autre part à la possibilité de mettre à profit la dynamique actuelle du monde des médias pour consolider l'entreprise propre au moyen d'une stratégie intelligente.

En ce qui concerne précisément le Bulletin des médecins suisses (BMS), l'auteur avait déjà recouru à une métaphore météorologique ici l'an dernier. «Le BMS veut affronter des vents contraires" s'intitulait l'article exposant la conjoncture économique de plus en plus difficile. Ce message est toujours vrai. Mais le «vaisseau amiral», pour reprendre le surnom occasionnel du BMS, est-il sur la bonne voie? Mène-t-il son fret à bon port? Remplit-il sa mission d'organe officiel de la FMH et de plateforme de discussion libre pour le corps médical et les autres cercles impliqués dans la santé? Depuis cette année, nous pouvons répondre de manière plus fiable à ces questions. Contrairement à sa première version de 2010, le sondage des lecteurs «Presse médicale 2017» réalisé cet été par le prestigieux bureau d'études de marché et de recherche sociale gfs-zürich fournit également des données qualitatives détaillées sur les 48 revues examinées [3]. Avec 2550 médecins participants, la taille de l'échantillon permet d'obtenir des réponses valides à une multitude de questions spécifiques. Il n'est pas présomptueux d'affirmer que les résultats du BMS n'ont pas à rougir.

Comme en 2010, le BMS reste la revue médicale la plus lue en Suisse en 2017. 94\% des médecins la lisent au moins occasionnellement ou la feuillettent (lectorat le plus vaste) et pas moins de $60 \%$ des médecins peuvent être touchés avec chaque édition (portée). Ces valeurs doivent impérativement être relevées pour qu'un sondage réponde aux critères de qualité de la REMP [4] qui constitue la référence dans les études des médias suisses. Mais elles ne fournissent aucune indication sur les rubriques lues - après tout, elles pourraient se limiter aux offres d'emploi ...

Ce n'est pas le cas. D’après le sondage, 2300 lectrices et lecteurs dévorent chaque "Et encore», c.-à-d. cette chronique. Si l'on ajoute les lecteurs réguliers et occasionnels, on arrive à près de 19000 . Et celles et ceux qui s'imaginent que cela place cette rubrique en tête des centres d'intérêt des lecteurs se trompent. La FMH a de bonnes raisons de se réjouir: son éditorial et les informations sur la politique professionnelle touchent environ 5000 lecteurs fidèles et jusqu'à 29000 lecteurs réguliers et occasionnels au total. Les points de vue, ainsi que les reportages et les interviews en seconde partie du BMS obtiennent des scores similaires. Fait particulièrement réjouissant, aucune rubrique n'est totalement ignorée, même la "partie culturelle» touche plus de 1000 lecteurs réguliers et quelque 16000 lecteurs occasionnels. $80 \%$ des sondés à peu près sont satisfaits du mix thématique du BMS, un résultat en phase avec ce comportement d'utilisation. Le lectorat est fortement attaché à la liberté d'expression et juge à $85 \%$ qu'il est très important que le BMS soit ouvert aux articles de toute sorte.

La rédaction se réjouit notamment que notre revue dédiée à la politique professionnelle et de la santé ne soit pas délaissée par les jeunes ( 35 ans). Nous avons néanmoins décidé de devenir encore plus attrayants pour ce groupe cible, y compris au travers de fonctionnalités supplémentaires sur Internet et, depuis peu, d'une page Facebook.

Bref, le BMS est sur la bonne voie, mais nous ne voulons pas nous reposer sur des lauriers imaginaires. Je vous souhaite, chères lectrices, chers lecteurs, de joyeuses fêtes et une excellente année 2018.

\section{Références}

1 Kurt H. Les éditions EMH fêtent leurs 20 ans. Bulletin des médecins suisses. 2017;98(51-52):1732-1734.

2 Scholer M. Des nuages sombres et du ciel bleu. Bulletin des médecins suisses. 2017;98(50):1693-1695.

3 Vous trouverez une synthèse des principaux résultats de l'enquête sur le site Web du BMS www.saez.ch $\rightarrow$ Tour d'horizon $\rightarrow$ Sondage des lecteurs gfs.

4 REMP signifie Recherches et études des médias publicitaires. La tâche de la REMP est de recenser systématiquement, en continu et objectivement l'usage qui est fait des médias. 